\title{
85672 - ALZHEIMER, SEXUALIDADE E INSTITUCIONALIZAÇÃO: UMA REVISÃO INTEGRATIVA
}

\author{
Pôster - Gerontologia
}

Tatiane Rocha Razeira / Razeira, TR / PUCRS; Maria Gabriela Valle Gottlieb / Gottlieb, MGV / PUCRS

Introdução: O processo de envelhecimento produz transformações, mudanças e adaptações na vida das pessoas em diferentes aspectos. Objetivo: analisar a produção científica sobre a sexualidade de pessoas idosas portadoras de Alzheimer e institucionalizadas. Método: revisão integrativa de artigos publicados nos últimos 10 anos, realizada na SCIELO, LILACS e PUBMED sendo utilizados os descritores (DECs): "sexuality", "Alzheimer disease", "institucionalization" e "sexuality", "dementia", "institucionalization". Resultados: Foram encontrados 6 artigos, dos quais 2 duplicados, restando 4 artigos, publicados entre 2014 e 2017. A necessidade da construção de conhecimentos acerca das temáticas na formação dos profissionais em saúde torna-se um aspecto contribuinte para uma postura cautelosa desses profissionais em relação à expressão da sexualidade das pessoas com Alzheimer e uma questão eticamente compassiva para quem cuida ${ }^{1}$. Quando se deparam com uma situação de expressão da sexualidade por parte dos residentes, a reação mais comum é buscar o aconselhamento de outros profissionais ${ }^{2}$. Visto que muitos profissionais entendem certas manifestações como inadequadas e não sabem como lidar com tais situações ${ }^{3}$. As pessoas idosas residentes expressam sua sexualidade de várias formas, e possuem atitudes positivas em relação à sexualidade, enfatizam o caráter individual tanto do interesse quanto da expressão sexual4. Conclusões: Observa-se escassez de publicações e é preciso desenvolver as temáticas na formação para que os profissionais compreendam e atendam as perspectivas e necessidades das pessoas idosas, para que essas possam manifestar sua sexualidade sem culpabilidade, com possibilidades e fluidez.

Palavras-chave: Sexualidade; Alzheimer; Institucionalização.

Referências: 1. Mahieu L., et al. Intimacy and Sexuality in Institutionalized Dementia Care: Clinical-Ethical Considerations. Health Care Anal. 2017; 25 (1): 52-71. 2. Villar F., et al. Staff attitudes towards sexual relationships among institutionalized people with dementia: does an extreme cautionary stance predominate? Int. Psych. 2014; 26 (3): 403-12. 3. Torrisi, M.,et al. Inappropriate behaviors and hypersexuality in individuals with dementia: An overview of a neglected issue. Geriat. Geront. Int. 2017; 17 (6): 865-874. 4. Mahieu L, Gastmans C. Older residents‘ perspectives on aged sexuality in institutionalized elderly care: a systematic literature review. Int. J. Nurs Stud., 2015; 52 (12): 1891-905. 